\title{
Assessment of Drug-related Problems in Patients with Chronic Diseases through Health Status Survey in a South Indian Rural Community Setting
}

\author{
R. ADEPU* AND P. K. ADUSUMILLI
}

Department of Pharmacy Practice, JSS College of Pharmacy, JSS University, Mysore to Bangalore Main Road, Near Fire Station, Sri Shivarathreeshwara Nagar, Mysore-570 015, India

Adepu and Adusumilli: Drug-related Problems in Patients with Chronic Diseases in South India

\begin{abstract}
A prospective study has been carried out to assess the incidence, prevalence and cost implications of drugrelated problems in patients with chronic diseases dwelling in a south Indian rural community setting. Details of patients with chronic diseases were recorded in a suitably designed data collection form and assessed for the potential drug-related problems in the prescriptions with the patient's consent. Hepler and Strand classification was used to categorize the identified drug related problems. Each drug-related problem was assessed for its significance level and graded either as minor, moderate or major based on expected clinical outcome. Cost implications due to drug-related problems were also evaluated. During the study period, the research pharmacist visited 550 houses and interviewed 2370 people. Among the interviewed individuals, $215(8.36 \%)$ people were found with chronic diseases. A total of 90 drug-related problems were observed in the patients' prescriptions who met the study criteria. Noncompliance (22), untreated indications (18) and drug interactions (14) were the most commonly found drug-related problems. Among all drug-related problems, 9 problems were graded as major, 37 were moderate and 44 were minor in severity. It is estimated that, a sum of 8464950 rupees could have been saved through the pharmacist intervention.
\end{abstract}

Key words: Drug related problems, house survey, chronic diseases, pharmacist intervention

A drug-related problem (DRP) is defined as an event or circumstance that actually or potentially interferes with desired health outcomes. DRPs can lead to ineffective pharmacotherapy and may become responsible for drug-related morbidity and mortality ${ }^{[1]}$. In modern clinical practice, prescribers often use more number of drugs to address the comorbidities in patients; and this situation further complicates when patients visit physicians of other specialities and may lead to potential drug related problems. Studies evaluating the prescriptions for elderly patients have observed frequent polypharmacy in the prescriptions and use of excessive or potential harmful drugs. Prescriptions containing multiple medications not only increase complexity of therapy regimen but also make the patient poorly adherent to the prescribed medication ${ }^{[2]}$.

This is an open access article distributed under terms of the Creative Commons Attribution-NonCommercial-ShareAlike 3.0 License, which allows other the remix, tweak, and build up to the non-commercially, as long as the author is credited and the new creations are licensed under the identical terms.

Accepted 02 July 2016

Revised 30 June 2016

Received 01 December 2014

Indian J Pharm Sci 2016;78(4):537-541 
Often doctors are also unaware about the medications the patients are taking ${ }^{[3]}$. Many patients also hesitate to disclose the use of other alternative therapies to their prescribers. The likely hood of herb-drug interactions is same or high as drug-drug interactions (DDIs) because herbal formulations contain mixtures of various plant constituents that are pharmacologically active ${ }^{[4]}$. Pharmacist's role is very much appreciated in hospital settings in identifying and resolving clinically important $\mathrm{DRPs}^{[5]}$. In community settings, patients with chronic diseases with comorbidities often take more medicines including over-the-counter (OTC) drugs. Such patients are prone to have more chances of experiencing DRPs in their prescriptions. If such DRPs are analysed and resolved, the therapeutic outcomes will be much better and saves the unwanted drug expenditure ${ }^{[6]}$. The present study is designed to assess the incidence and severity of DRPs precipitated in prescriptions received by community patients with chronic diseases, dwelling in community. Further, an assessment was also made to analyse the economic impact of the DRPs.

The present study was a prospective study, conducted in the selected rural communities of south India, over a period of nine months from June 2012 to February 2013. Patients of either sex suffering from chronic diseases willing to give consent were included in the study. Acutely ill patients were excluded from the study. The study was approved by the Institutional Human Ethics Committee of JSS College of Pharmacy, Mysore. The study pharmacist conducted house visits and reviewed prescriptions and physician notes of patients with chronic diseases. Collected data was documented in the suitably designed data collection form. DRPs identified in prescriptions were categorized based on Hepler and Strand classification. Each DRP was assessed for its level of significance and was rated either as minor, moderate or major based on its threat to the patient.

During the study period, about 550 houses were visited in different rural localities of Mysore city and a total of 2370 people (1170 men, 1200 women) were interviewed. Among them, 215 (105 men, 110 women) patients were identified with chronic diseases and they were interviewed.

Number of patients suffering from chronic diseases with ICD classification is presented in Table 1. Prevalence of the chronic diseases is calculated by using the following formula. Percentage prevalence of the chronic diseases $=($ No. of people identified with chronic
TABLE 1: ICD 10 CLASSIFICATION OF THE CHRONIC DISEASES AMONG THE PATIENTS

\begin{tabular}{lcc}
\hline Chronic disease condition & ICD code & $\begin{array}{c}\text { Number of } \\
\text { patients } \\
\text { identified }\end{array}$ \\
\hline Hypertension & I 10 & 89 \\
Diabetes Mellitus & E 10 & 45 \\
Asthma & J 45 & 10 \\
Arthritis & M 13 & 04 \\
Gastritis & K 29 & 03 \\
Epilepsy & G 41 & 03 \\
Migraine & G 43 & 02 \\
Depression & F 32 & 02 \\
Hypothyroidism & E 03 & 02 \\
Osteoarthritis & M 15 & 01 \\
Tuberculosis & A 15 & 01 \\
Hypertension+Diabetes Mel- & I 10 + E 10 & 42 \\
litus & I 10 + E 78 & 03 \\
Hypertension+Hyperlipidemia & F 32 + M 13 & 02 \\
Depression+Arthritis & I 10 + E 10 + & 02 \\
Hypertension+Diabetes & E 03 & \\
mellitus+Hypothyroidism & J 45 + E 03 & 01 \\
Asthma + Hypothyroidism & I 10 + F 32 & 01 \\
Hypertension + Depression & & \\
\hline
\end{tabular}

$\mathrm{N}=215$

diseases/total number of people interviewed) $\times 100$. The prevalence of the chronic diseases among the individual interviewed was found as $9.07 \%$.

Hepler and Strand categorized DRPs into eight categories as over dose, sub-therapeutic dose, failure to receive drugs, improper drug selection, drug interaction, adverse drug reaction, drug use without indication, untreated indication.

A total of 90 DRPs (40 in men, 50 in women) were identified at a rate of $41.8 \%$. Of the 90 DRPs, 56 were observed in adults and 34 were observed in geriatric population. The most common DRP found was 'failure to receive drug', which was accounted for $24 \%(n=22)$ of total DRPs followed by untreated indication $20 \%$ $(n=18)$. Classification of DRPs is presented in Table 2. All the DRPs that led to the primary consultation and hospitalization was also calculated and presented in Table 3. Diseases involved in precipitating the DRPs were assessed and presented in Table 4. Among all the DRPs resulted, $10 \%$ of the DRPs were severe and $41 \%$ were moderate resulting in primary consultation and hospitalization. Results are presented in fig. 1.

The following mathematical formula was applied to calculate societal cost savings in the study, Number of identified DRPs $\times(\%$ avoided primary care visits $\times$ societal cost/visit $)+(\%$ avoided hospitalizations $\times$ societal cost per hospitalization day $\times$ number of days). Societal cost saving of patients 
TABLE 2: CLASSIFICATION OF DRUG-RELATED PROBLEMS

\begin{tabular}{lc}
\hline Type of DRPs & Number of DRPs (\%) \\
\hline Untreated indication & $18(20)$ \\
Drug use without indication & $05(06)$ \\
Improper drug selection & $01(01)$ \\
Sub-therapeutic dosage & $13(14)$ \\
Over dosage & $09(10)$ \\
Failure to receive drugs & $22(24)$ \\
Drug interaction & $14(16)$ \\
Adverse drug reactions & $08(09)$ \\
\hline
\end{tabular}

DRPs=Drug-related problems
TABLE 3: CONSEQUENCE OF DRUG-RELATED PROBLEMS

\begin{tabular}{lc}
\hline DRPs led to & Number of DRPs (\%) \\
\hline Primacy care visit & $46(51)$ \\
Hospitalisations & $31(35)$ \\
No influence & $13(14)$ \\
\hline
\end{tabular}

DRPs=Drug-related problems

TABLE 4: DISEASES INVOLVED IN DRUG-RELATED PROBLEMS

\begin{tabular}{|c|c|c|}
\hline Types of DRPs & Disease diagnosed & Number of incidences \\
\hline \multirow[t]{6}{*}{ Drug interactions(14) } & Hypertension & 8 \\
\hline & Diabetes Mellitus & 1 \\
\hline & HTN+DM+Hypothyroidism & 1 \\
\hline & Epilepsy & 1 \\
\hline & Asthma+HTN & 2 \\
\hline & Asthma+Hypothyroidism & 1 \\
\hline \multirow[t]{6}{*}{ Adverse drug reactions (8) } & Depression & 1 \\
\hline & $\mathrm{DM}+\mathrm{HTN}$ & 2 \\
\hline & Depression+HTN & 1 \\
\hline & Migraine & 1 \\
\hline & Hypertension & 2 \\
\hline & Arthritis+Depression & 1 \\
\hline \multirow[t]{6}{*}{ Over dosage (9) } & Gastritis & 2 \\
\hline & Hypertension & 2 \\
\hline & Hypothyroidism & 1 \\
\hline & $\mathrm{DM}+\mathrm{HTN}$ & 2 \\
\hline & HTN+Dyspepsia & 1 \\
\hline & Arthritis & 1 \\
\hline \multirow[t]{4}{*}{ Untreated indication (18) } & HTN & 11 \\
\hline & DM & 5 \\
\hline & Hyperlipidemia & 1 \\
\hline & Migraine & 1 \\
\hline \multirow[t]{5}{*}{ Sub-therapeutic dosage (13) } & HTN & 6 \\
\hline & $\mathrm{DM}$ & 3 \\
\hline & $\mathrm{HTN}+\mathrm{DM}$ & 2 \\
\hline & Asthma & 1 \\
\hline & Arthritis & 1 \\
\hline Improper drug selection (1) & $\mathrm{DM}$ & 1 \\
\hline \multirow{2}{*}{ Drug use without indication (5) } & HTN & 3 \\
\hline & $\mathrm{HTN}+\mathrm{DM}$ & 2 \\
\hline \multirow[t]{4}{*}{ Failure to receive drugs (22) } & HTN & 9 \\
\hline & Asthma & 2 \\
\hline & & 7 \\
\hline & $\mathrm{HTN}+\mathrm{DM}$ & 4 \\
\hline
\end{tabular}

DRPs=Drug-related problems, HTN=Hypertension, DM=Diabetes mellitus

under evaluation in this study was found to be Rs. 84 64950 .

The study provides data on extent of DRPs observed in patients suffering from chronic diseases in community settings of Mysore city. The results of this study provide information which may be used in designing measures to minimize DRPs in patients with chronic diseases in community. Such corrective measures not only improve quality use of medicines but also improve quality of life.

The incidence of chronic diseases in our study was found to be $9.07 \%$. Among all chronic diseases, hypertension is the major chronic disease with which people are suffering. Health awareness programs and camps may be organized for the public in community to minimize the incidence and prevalence of chronic 


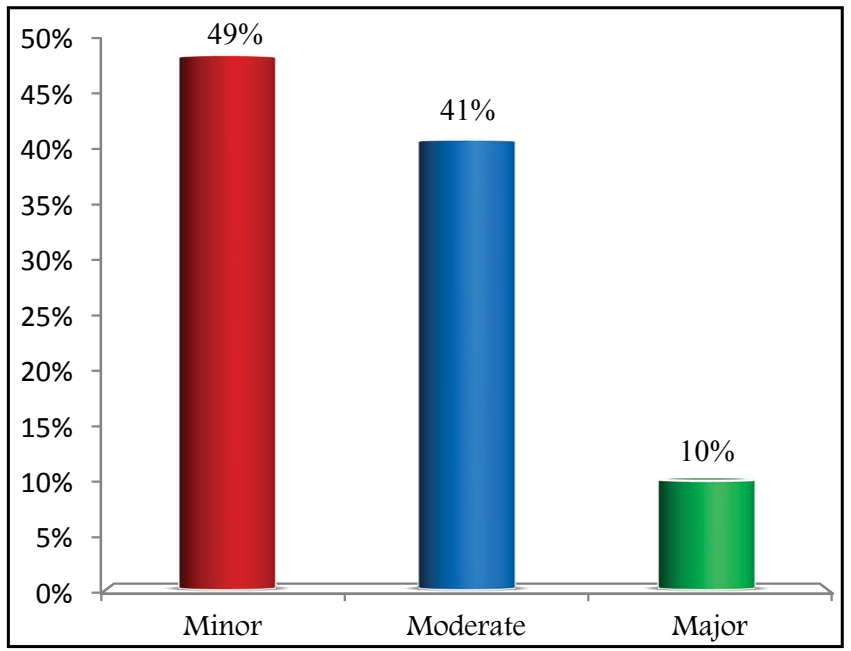

Fig. 1: Significance level of drug-related problems.

-m- Minor, -"- Moderate, -๘- Major.

diseases. In a global chronic prevalence study, it was observed that the incidence and prevalence of chronic diseases is progressively increasing worldwide at a rate between 16-44\%. Major factors for this incidence are age, modern life styles and food habits ${ }^{[7]}$. Although the incidence is less in our study, it may be due to the majority data collected was from rural and semi urban area. In addition to this, the cultural balance of the population in rural area may be another contributing factor for fewer incidences.

During the study period, 90 DRPs were observed in a total of 215 patients with a prevalence rate of $41.8 \%$. In the study carried out by Paulino et al. ${ }^{[8]}$ where the prevalence of DRPs was reported to be $63.7 \%$. The incidence rate in our study was found lower compared to the above study. This may be because of poor disclosure of health related information by patients to the study pharmacist and also the rural living conditions.

Among 90 DRPs identified during the study period, male patients who had DRPs are $44.5 \%(n=40)$ and females were $55.5 \%(n=50)$. There is predominance of gender for having DRP between males and females. In our study female population were found more prone to having DRP, this observation is contrasted with the demographic reports of the study conducted by Koh et al. who cited there is no predominance of gender for having DRPs ${ }^{[9]}$.

The prevalence of DRPs were $40.6 \%$ in geriatric patients and $43.6 \%$ in adults, it is observed from the study that there is no much significance in age group for having DRPs. Among the potential contributing factors of DRPs, the association between age and the incidence of DRPs has much connotation and it is well documented in a study conducted by Lau and Dolovich, which revealed that geriatric patients are enduring DRPs at a rate of $62.8 \%$ which is found to be higher compared to present study ${ }^{[10]}$.

Most of the DRPs observed in our study were due to failure to receive drugs $(24.0 \%)$. It was followed by untreated indication $(20.0 \%)$ and DDIs (16.0\%). This observation is supported with the study conducted by Koh et al. ${ }^{[9]}$ in which noncompliance and DDIs accounted for a substantial amount of potential DRPs. The reason for these DRPs in high number in our study may be due to inadequate understanding about the disease and medications among the patients. Poor economic status of patients from rural area contributed for failure to receive the drugs.

The causality of adverse drug reactions (ADRs) identified was assessed and documented. Majority (62.5\%) of the ADRs belonged to 'probable' category on WHO scale. Half $(50.0 \%)$ of the observed ADRs were 'mild' in severity and rest were 'moderate' and none of the ADRs were found to be 'severe'. Amongst the identified ADRs, $67.5 \%$ were 'not preventable' and $8.54 \%$ of them were 'probably preventable' and $20 \%$ were 'definitely preventable'. Often suspected ADRs are not reported adequately by prescribing doctors it may be due to heavy patient load and non-mandatory reporting system.

In our study, it was observed that patients were inadequately informed about safe use of their medications, so patients were provided with information regarding the use of their medications to improve the adherence behavior.

All identified drug interactions were classified into major, moderate and minor. DDIs were implicated in $16 \%$ of the DRPs. Drug interaction between aspirin and losartan belongs to 'major' in severity level and may cause elevation of serum $\mathrm{K}^{+}$levels. So patient should be monitored with serum potassium levels. The interaction between aspirin and furosemide belonged to 'moderate' in severity level and many of the drug interactions belongs to class 'minor' in severity level which can be managed easily and some are set to be theoretical in consideration.

DRPs identified were preventable in majority of cases i.e. diclofenac is used as analgesic where aceclofenac is taken by patient at same time. This DRP was categorized into over-usage of medication which could have been prevented if a proper history of patient had 
been obtained.

Untreated indications were causing $20 \%$ of total DRPs. Hypertension was the main health related issue that was left untreated in the study patients. In majority of the cases, the issues were identified by study pharmacist and brought to the notice of patients and were directed them to consult their physicians for further evaluation and needful action.

Sub-therapeutic dose was indicated in $14 \%$ of total DRPs. In one such instance involving sub-therapeutic dose, a patient receiving 15 units of insulin mixtard since 18 months, and on examining patient's fasting blood glucose levels, it was found higher than normal levels $(140 \mathrm{mg} / \mathrm{dl})$. This is because the dose given was not adequate for the patient to keep his glucose levels under control. This type of incidences happen as patients do not have the regular follow ups in the primary care settings.

DRPs identified were assessed and rated as minor, moderate and major. The percentage of 'major' DRPs $(10 \%)$ identified was lower than the study finding observed by Alderman ${ }^{[11]}$ in which 'major' class of DRPs were $20 \%$. The difference in the number between the two studies is due to lack of detailed availability of patients condition, as mentioned study was conducted in a well-established hospital where as present study was done in a rural community settings.

An instance of a DRP categorized as 'major' where patient was receiving venlafaxine for the depression. The patient was experiencing dizziness as an ADR and the patient was supposed to be confined to bed if it continues. This ADR was identified and brought to notice of the concerned physician and also educated the patient about the adverse effect of drug and also the strategy to overcome.

An instance of DRP categorized as moderate where a patient was taking pantoprazole for longer period than prescribed duration, the research pharmacist after assessing the problem, advised the patient for cessation of the drug. This intervention resulted in reduction of cost of medication usage and also ensured the safe use of drugs.

DRP are known to cause hospitalization, increased suffering and decreased quality of life and increased health care expenditures. After identifying and assessing a DRP, the expected clinical outcome in terms of improved therapeutic effect and direct and indirect cost implication was hypothetically calculated and the net financial burden to the society was demonstrated. In our study we found that the net financial burden due to DRPs was Rs. 8464 950. This amount would have been saved if adequate intervention was made and prevented the DRPs.

According to a study conducted by Westerlund and Marklund, roughly 64 million prescriptions are filled each year in Sweden. If DRPs were identified resolved at the same rate, a total of $€ 358$ million can be saved when extrapolated at national level ${ }^{[12]}$. Similarly in our study, the findings are extrapolated to the entire Mysore city population the net societal savings may be projected as Rs. 3413975 600. The above findings in both studies suggest the importance of pharmacist interventions in minimizing the DRPs.

DRPs may occur in all stages of the medication process starting from prescribing to dispensing stage. Lack of follow-ups and reassessment of medical treatment also contribute to DRPs ${ }^{[13,14]}$. Pharmaceutical care is a cooperative activity between health care professionals provided directly to the patient and adds to the effectiveness of improving the quality of patient care. Pharmaceutical care identifies and resolves actual or potential DRP ${ }^{[15]}$.

The prevalence of chronic diseases in the study people was observed to be $9.07 \%$. The incidence of DRPs in the study population was observed at a rate of $41.8 \%$. The most common DRP identified in study was failure to receive drugs $24 \%(\mathrm{n}=22)$ followed by untreated indication $20 \%(\mathrm{n}=18)$. Female population was at a higher risk for having DRPs compared to male patients.

Pharmacist intervention will decrease the incidence of DRPs in patients with chronic diseases and comorbidities. Such service will increase the net societal savings and provides a better patient care.

\section{Acknowledgements:}

Authors would like to thank JSS University, Principal, JSS College of Pharmacy, and Head, Department of Pharmacy Practice for their continuous support.

\section{Financial support and sponsorship:}

Nil.

\section{Conflicts of interest:}

There are no conflicts of interest. 